\title{
ANÁLISIS DEL BIENESTAR PSICOLÓGICO, ESTADO DE SALUD PERCIBIDO Y CALIDAD DE VIDA EN PERSONAS ADULTAS MAYORES
}

\section{ANALYSIS OF PSYCHOLOGICAL WELL-BEING, PERCEIVED HEALTH STATUS AND QUALITY OF LIFE IN OLDER ADULTS}

\section{ANÁLISE DE BEM-ESTAR PSICOLÓGICO, ESTADO DE SAÚDE E QUALIDADE DE VIDA EM ADULTOS MAIS VELHOS}

\author{
Ángel De-Juanas Oliva \\ Universidad Nacional de Educación a Distancia, España \\ María Rosario Limón Mendizábal \\ Universidad Complutense de Madrid, España \\ Enrique Navarro Asencio \\ UniVERSIDAd INTERNACIONAL DE LA RIOJA. ESPAÑA
}

RESUMEN: En el marco de la intervención educativa en contextos sociales para la mejora de la calidad de vida de las personas adultas mayores, describir el estado en el que se encuentran estas personas debe ser un objetivo prioritario para promocionar un adecuado envejecimiento activo y saludable. Por ello, el propósito de este estudio fue analizar, en una muestra de 328 participantes de la Comunidad de Madrid, la asociación entre el bienestar psicológico, estado de salud percibida y hábitos saludables que se encuentran directamente relacionados con la calidad de vida de los mayores. Para ello, se aplicó una encuesta elaborada ad hoc y la Escala de Bienestar Psicológico de Carol Ryff que establece un modelo que atiende a seis dimensiones: Autoaceptación, Relaciones positivas con otras personas, Autonomía,
Dominio del entorno, Propósito de vida y Crecimiento personal. Los resultados obtenidos muestran la tendencia a que las personas que afirman que su estado de salud ha sido muy malo tienden a presentar resultados más altos en Autoaceptación y Propósito de vida que aquellos que lo consideran bueno o muy bueno. Asimismo aquellos que durante su tiempo libre realizan el ejercicio físico que desean obtienen puntuaciones altas en la escala de Autoaceptación. En este sentido, los que realizan ejercicio regularmente obtienen puntuaciones más altas en Autoaceptación y Dominio del entorno. Finalmente, los mayores que no salen con otras personas, tienen menos posibilidades de hablar con otras personas sobre sus problemas, se distraen menos de lo que desean y reciben menos elogios obtienen puntuaciones más bajas 
para la práctica totalidad de las dimensiones de bienestar psicológico.

PALABRAS CLAVE: educación de adultos, envejecimiento, bienestar psicológico, salud, relaciones interpersonales.

ABSTRACT: As part of the educational intervention in social contexts to improve the quality of life of elderly people, to describe the state of this kind of people should be a priority to promote proper active and healthy aging. Therefore, the aim of this study was to analyze, in a sample of 328 participants of Madrid region, the association between psychological welfare, health status perceived and health habits that are directly related to the quality in this part of the life. For this purpose, an ad hoc survey was applied together with the Psychological Well-being Scale by Carol Ryff which sets a model with six dimensions: Self-acceptance, Positive relations with others, Autonomy, Environmental mastery, Purpose in life and Personal growth. The results show a tendency for people who say that their health is very poor, they tend to score higher on Self-acceptance and Purpose of life than those who consider their health as good or very good. Also those who do exercise during their free time get high scores on the scale of Selfacceptance. In this sense, those who do exercise regularly score higher on Self-acceptance and Environmental mastery. Finally, elderly people who do not go with other people are less likely to talk to other people about their problems and receive less praise. They get lower scores for almost all of the dimensions of psychological welfare.

KEYWORDS: adult education, ageing, psychological welfare, health, interpersonal relations.
RESUMO: Como parte da intervenção educativa em contextos sociais para melhorar a qualidade de vida das pessoas idosas, para descrever o estado deste tipo de pessoas deve ser uma prioridade para promover o envelhecimento ativo e saudável adequada. Portanto, o objetivo deste estudo foi analisar, em uma amostra de 328 participantes da região de Madrid, a associação entre o bem-estar psicológico, estado de saúde e hábitos de saúde que estão diretamente relacionados com a qualidade nessa parte da vida. Para este efeito, uma pesquisa ad hoc foi aplicado juntamente com o bem-estar psicológico Scale por Carol Ryff, que estabelece um modelo com seis dimensões: autoaceitação, relações positivas com os outros, autonomia, domínio do ambiente, propósito na vida e crescimento pessoal. Os resultados mostram uma tendência para as pessoas que dizem que sua saúde é muito pobre, eles tendem a pontuação mais elevada em auto-aceitação e finalidade da vida do que aqueles que consideram sua saúde como boa ou muito boa. Também aqueles que fazem exercício durante seu tempo livre obter pontuações mais altas na escala de auto-aceitação. Neste sentido, aqueles que fazem exercício regularmente pontuação maior na auto-aceitação e domínio ambiental. Finalmente, as pessoas idosas que não vão com outras pessoas são menos propensos a falar com outras pessoas sobre seus problemas e receber menos elogios. Eles recebem pontuações mais baixas para quase todas as dimensões do bem-estar psicológico.

PALAVRAS-CHAVE: educação de adultos, bemestar psicológico de envelhecimento, saúde, relações interpessoais.

[ 154 ] ÁNGEL DE-JUANAS OLIVA, MARÍA ROSARIO LIMÓN MENDIZÁBAL, ENRIQUE NAVARRO ASENSIO SIPS - PEDAGOGIA SOCIAL. REVISTA INTERUNIVERSITARIA [1139-1723 (2013) 22, 153-168] TERCERA ÉPOCA 


\section{Introducción}

El presente trabajo ${ }^{1}$ tiene en consideración que uno de los logros más significativos de nuestro tiempo es la prolongación de la vida de las personas, sin embargo, esta prolongación no tendría sentido si no se consigue paralelamente una mejora de la calidad de vida. Esta fue definida en 1994 por el grupo de la Calidad de Vida de la OMS (WHOQOL) ${ }^{2}$, como una percepción individual de la posición en la vida, en el contexto del sistema cultural y de los valores en que vive la gente y relacionada con sus objetivos, expectativas y preocupaciones. Se trata de un concepto amplio, subjetivo, que incluye de forma compleja la salud física de la persona, su estado psicológico, el nivel de independencia, las relaciones sociales, las creencias y las convicciones personales y su relación con aspectos importantes del medio ambiente (OMS, 2001).

Fernández Ballesteros (1992) enumera alguno de los elementos que contribuyen a la calidad de vida. Por una parte señala dimensiones personales como las relaciones sociales, la satisfacción, las actividades de ocio, la salud y las habilidades funcionales; y por otra parte, señala dimensiones socioambientales, entre las que distingue el apoyo social, las condiciones económicas, los servicios de salud y sociales, calidad del ambiente y factores culturales.

En opinión de varios autores (Brown, 2000, Wolkenstein y Butler, 1992 cit. por Schalock y Verdurgo, 2003), se puede decir que los diferentes conceptos de la calidad de vida en las personas mayores se caracterizan por un aspecto relevante, su carácter multidimensional, que tiene en cuenta no solo el funcionamiento físico, la energía y vitalidad personal, sino también el bienestar psicológico y emocional, la ausencia de problemas de comportamiento, el funcionamiento social y el sexual, el apoyo recibido y percibido, junto con la satisfacción con la vida y la percepción del estado de salud (Gonçalves, 2012, p. 116).

Por otro lado, como resultado de la transición de altas a bajas tasas de fecundidad y la reducción continua de la mortalidad, se ha producido un incremento de la proporción de personas mayores de 65 años que, según el Fondo de Población de las Naciones Unidad (UNFPA, 2011), se prevé que sea del $22 \%$ en 2050 . Este hecho se vive, por sus posibles repercusiones, como una preocupación en cada uno de los aspectos de la vida sobre el nivel individual, comunitario, nacional e internacional así como en las facetas sociales, económicas, políticas, culturales, psicológicas y espirituales. No obstante, el hecho de que aumente la esperanza de vida en la mayor parte del mundo, debe considerarse un logro de la humanidad (Rubio, 2012, p. 23).

En este sentido, la Comisión para el Estudio de los Efectos sobre el Envejecimiento en el futuro de la Sociedad del Bienestar (IMSERSO, 2010) afirma que los mayores serán determinantes en el mantenimiento de las redes sociales familiares y en la articulación de las generaciones, así como una potencial fuente de consejo.

Este aumento de la población mayor hace necesario analizar y comprender mejor su significado. Por esta razón, desde hace más de tres décadas se han ido sucediendo encuentros científicos con el fin de analizar el envejecimiento de la población, sus consecuencias y los retos que todo ello plantea. Entre los retos que se presentan, y en relación directa con el desarrollo de este trabajo, está el fomento de la salud y el bienestar en la vejez, también el cuidado que necesitan las personas que envejecen, potencialmente dependientes, los beneficios psicosociales de la actividad física, la calidad de vida, la influencia de los estilos de vida en las imágenes y actitudes frente al envejecimiento, la educación y la formación en esta etapa de la vida, etc.

Estos elementos han sido abordados en la Primera y la Segunda Asamblea Mundial sobre el Envejecimiento, celebradas en Viena (1982) y en Madrid (2002), respectivamente, que dieron lugar a la génesis de Planes de Acción Internacionales sobre el envejecimiento que todavía hoy constituyen un referente e inspiran el diseño de políticas y actuaciones a diversos niveles (Limón, 2011).

De tal modo, en la Segunda Asamblea Mundial sobre el Envejecimiento, en su declaración final, se 
plantea que "la participación en actividades sociales, económicas, culturales, deportivas, recreativas y de voluntariado contribuye también a aumentar y mantener el bienestar personal" y, consecuentemente, como recomendación "hay que alentar a las personas de edad a que mantengan o adopten modos de vida activos y saludables que incluyan actividades físicas y deportes".

Las recomendaciones de la asamblea citada nos acercan a los enfoques que deben tener las actividades que se proponen en diferentes foros. Estas recomendaciones suponen una intervención transversal al trabajar con enfoques de diferentes ámbitos (Montiel y Merino, 2011). Es decir, esta diversidad temática afecta a la salud, el comportamiento, la relación social y la motricidad de la persona mayor en sus beneficios y expectativas, que se combinan de manera integral para explicar los beneficios de la actividad física en la vejez y llegar a una longevidad activa.

\section{Longevidad y bienestar psicológico}

El envejecimiento saludable es el resultado de un proceso que dura toda la vida. Por consiguiente, es necesario optimizar el desarrollo del individuo desde la primera infancia. Sabemos que una serie de factores en la primera infancia, la adolescencia, durante los principios de la edad adulta y durante la madurez, así como la situación vital actual de las personas mayores, determinan el proceso de envejecimiento y bienestar en la vejez. El envejecimiento saludable es un reto para todos los gerontólogos y geriatras, un reto para los científicos de muchas disciplinas, un reto para los políticos y un reto para todos aquellos que trabajamos con las personas mayores.

Los determinantes ambientales, los estilos de vida y el marco en el que se vive son fundamentales para la prevención de determinadas enfermedades y para envejecer con éxito. Las medidas de prevención para mantener y aumentar las capacidades son necesarias.

Mora (2009, p. 17) apunta doce claves, para envejecer con éxito: restricción calórica y hábitos dietéticos saludables; ejercicio físico aeróbico; ejercicio mental; viajar; adaptarse a los cambios sociales; no vivir solo; no fumar; no sufrir estrés con desesperanza; tener un buen sueño; evitar el "apagón emocional"; dar sentido a la vida con agradecimiento; y alcanzar la felicidad, entendida ésta como ese punto de felicidad que se consigue cuando se está en el mundo sin necesidad de él.

En otro sentido, Lehr (2008) señala que por muy influyentes que sean los factores de tipo genético, biológico y físico, no son suficientes para explicar la longevidad. Los resultados de la investigación internacional en esta materia indican una serie de relaciones interesantes, y puntualiza que "[...] se debería hacer hincapié sobre todo en la idea que una serie de factores susceptibles de influir en una mayor esperanza de vida interactúan unos con otros" (p. 246). A su vez, Lehr enuncia una serie de factores asociados a una supervivencia más larga. Uno de estos factores es, precisamente, la existencia de estados de ánimo positivos, los contactos sociales, la actividad, el humor, las actividades físicas y el deporte, etc., que ponen de manifiesto la importancia de una educación para la salud y de una pedagogía del humor (Fernández y Limón, 2012), no sólo en la vejez sino en todas las etapas de la vida.

Por su parte, Rodríguez-Artalejo (2011, p. 2) considera que aunque se van acumulando evidencias de que el envejecimiento satisfactorio se incuba desde el vientre materno, hay unas cuantas recomendaciones útiles en la edad adulta y en la vejez, todas ellas relacionadas con hábitos saludables entre los que se encuentra la actividad física regular ajustada a la capacidad individual. Por último, señala este autor, que todo lo anterior será más fácil si se tiene una buena red social, a menudo imprescindible para recibir ayuda emocional y material.

Con todo ello, se pone de manifiesto la importancia del ejercicio físico, de las relaciones sociales, del ocio y de la formación, entre otros, en esta etapa de la vida potenciando una educación gerontológica para el bienestar psicológico y la calidad de vida de este sector de población.

[ 156 ] ÁNGEL DE-JUANAS OLIVA, MARÍA ROSARIO LIMÓN MENDIZÁBAL, ENRIQUE NAVARRO ASENSIO SIPS - PEDAGOGIA SOCIAL. REVISTA INTERUNIVERSITARIA [1139-1723 (2013) 22, 153-168] TERCERA ÉPOCA 
El constructo de bienestar psicológico se ha desarrollado sin precisión en diversas investigaciones (Ryan y Deci, 2001, Díaz et al., 2006, George, 2006).

Algunos expertos asocian el bienestar psicológico a la felicidad desde una perspectiva hedónica pero también se une al desarrollo de las capacidades humanas. En cualquiera de los casos, el bienestar psicológico se corresponde con un juicio subjetivo, global y relativamente estable de satisfacción con la vida y la moral de las personas (George, 2010). De acuerdo a este planteamiento, Ryff (1989a, 1989b) planteó un modelo multidimensional de bienestar psicológico que responde a seis dimensiones: Auto-aceptación; relaciones positivas con otras personas; autonomía; dominio del entorno; propósito en la vida; y crecimiento personal.

Conforme a este escenario teórico, el propósito de este trabajo consistió en analizar la influencia del estado de salud percibido y los hábitos saludables, que afirman llevar a cabo las personas adultas mayores, de acuerdo a su bienestar psicológico subjetivo percibido que atiende a las dimensiones de auto-aceptación, relaciones positivas, dominio del entorno, crecimiento personal, propósito en la vida y autonomía.

\section{Método}

Para lograr el objetivo establecido se llevó a cabo un estudio cuantitativo a partir de la información recogida con dos cuestionarios durante el segundo trimestre de 2011, empleando un diseño ex-post-facto. Por un lado, se realizó un análisis descriptivo de las variables implicadas y, por otro, un estudio correlacional mediante pruebas estadísticas no paramétricas para la comparación de grupos.

\subsection{Participantes}

La muestra estuvo formada por 328 participantes (30.5\% varones, 100; 69.5\% mujeres, 228) de Madrid capital. La selección de la misma fue incidental y atendió a criterios de acceso y disponibilidad. Los participantes del estudio pertenecieron a tres centros de día y cinco centros culturales ubicados en diferentes distritos de la ciudad. La tipología de los participantes responde a un modelo socioeconómico medio y medio-alto.

La edad de los participantes supera los 65 en la mayoría de los casos. Un 43.6\% (143 sujetos) se encuentran entre 66 y 75 años mientras que el 29.6 (97 sujetos) supera esa edad. El resto, un $26.8 \%$ ( 88 sujetos) tienen una edad inferior a los 66 años, si bien el participante de menor edad tenía 54 años. Respecto al estado civil, más de la mitad están casados (55.5\%), un $23 \%$ son viudos y el resto (un $14 \%$ ) están solteros.

\subsection{Procedimiento}

Se asistió a los centros para explicar a los directores, docentes y mayores el propósito del estudio, con el fin de que se concediera la autorización para la aplicación de los instrumentos de recogida de información. Los instrumentos fueron aplicados por los autores y colaboradores del estudio que fueron formados previamente en las directrices generales a seguir. En especial, en aquellas relativas a la finalidad del estudio, el trato con personas adultas mayores y la protección de los datos. La aplicación tuvo lugar en sesiones de una hora durante el horario de permanencia en el centro por parte de los participantes. Todos los sujetos de la muestra colaboraron de manera voluntaria y se garantizó el anonimato en las respuestas. Una vez recogidos los datos, se procedió a la codificación, ordenación y grabación informática de las respuestas a los instrumentos en una base de datos para su posterior tratamiento estadístico. 


\subsection{Instrumentos}

Los instrumentos utilizados para el estudio fueron, en primer lugar, un cuestionario elaborado ad hoc para conocer el estado de salud percibido de las personas mayores y sus hábitos saludables. Este instrumento contó con tres ítems que servían para recoger datos sobre variables sociodemográficas (sexo, edad y estado civil). A los que deben añadirse once reactivos que recogían información sobre: el estado de salud percibido; la realización de ejercicio físico de forma regular; si realizan todo el ejercicio físico que desearían en su tiempo libre; si reciben invitaciones para salir y distraerse con otras personas; si reciben elogios y reconocimientos cuando hacen bien las cosas y si tienen la posibilidad de hablar con alguien de sus problemas. Estos ítems fueron tomados como variables independientes en el estudio.

De estos ítems, siete estaban formulados en una escala valorativa comprendida entre 1 y 5, otros cuatro tenían un carácter dicotómico y ofrecían la alternativa de respuesta sí o no.

Para la realización de este instrumento se utilizaron, en su mayoría, reactivos de la Encuesta Nacional de Salud 3 (2006) y de la Encuesta Europea de Salud 4 (2009) realizada por el Instituto Nacional de Estadística y el Ministerio de Sanidad, Política Social e Igualdad de España.

En segundo lugar, se utilizó la Escala BP de Bienestar Psicológico de Ryff, versión adaptada en español por Díaz et al. (2006). Esta escala multidimensional es un instrumento que cuenta con 39 ítems en los que los participantes responden entre 1 (totalmente en desacuerdo) y 6 (totalmente de acuerdo). Se trata de una escala valorativa que atiende a criterios de evaluación subjetivos del buen funcionamiento psicológico sobre la base de seis dimensiones o atributos positivos del bienestar psicológico que establece Ryff (1989a, 1989b y 1995). Estas dimensiones se utilizaron como variables dependientes en el estudio.

La primera dimensión es la de Auto-aceptación o actitud positiva hacia el yo. Constituye uno de los criterios más relevantes del bienestar psicológico. Está asociado a la autoestima y conocimiento de uno mismo. La capacidad de sentirse satisfecho con uno mismo es necesaria aún reconociendo las propias limitaciones (Keyes, Ryff y Shmotkin, 2002).

La segunda dimensión es la de Relaciones positivas con otras personas. Se concreta en la capacidad que tiene la persona de mantener relaciones de confianza, estables y de intimidad.

La tercera dimensión es la de Autonomía. Se considera que las personas que presentan un funcionamiento psicológico positivo tratan de sustentar su propia individualidad en diversos contextos y situaciones mediante la capacidad de autodeterminación para mantener su independencia y su propia autoridad personal.

La cuarta dimensión es la denominada de Crecimiento personal. Para lograr el bienestar psicológico es preciso que las personas evolucionen, desarrollen sus potencialidades y sigan creciendo en base a aprendizajes positivos.

La quinta dimensión es la de Dominio del entorno. Supone que la persona considere que es buena en el manejo y control de las responsabilidades cotidianas. Esta dimensión se asocia al locus de control, a la auto-eficacia y a la capacidad de generar entornos favorables que permitan la satisfacción de los deseos y necesidades propias.

Finalmente, la sexta dimensión es la de Propósito de vida. Esta dimensión recoge ítems en los que se refleja el bienestar psicológico positivo de la persona sobre la base de la capacidad de marcarse metas, definir objetivos, estar motivados y otorgar a la vida un sentido.

Por otro lado, se examinó la consistencia interna del BP mediante el alfa de Cronbach. Los resultados obtenidos muestran un valor de .921 para el total de la escala. Este valor puede considerarse más que aceptable. No obstante, si tiene en consideración la fiabilidad para cada una de las dimensiones en comparación con los hallazgos de Díaz et al. (2006) para la versión española de la BP, se observan los siguientes resultados:

[ 158 ] ÁNGEL DE-JUANAS OLIVA, MARÍA ROSARIO LIMÓN MENDIZÁBAL, ENRIQUE NAVARRO ASENSIO SIPS - PEDAGOGIA SOCIAL. REVISTA INTERUNIVERSITARIA [1139-1723 (2013) 22, 153-168] TERCERA ÉPOCA 
Tabla 1. Comparativa de la consistencia interna de las dimensiones de la Escala BP para el estudio de Díez et al. (2006) y la presente investigación

\begin{tabular}{lccc}
\hline & & $\begin{array}{c}\text { Consistencia interna obtenida } \\
\text { por Díez el al. (2006) }\end{array}$ & $\begin{array}{c}\text { Consistencia interna obtenida } \\
\text { del presente estudio }\end{array}$ \\
\hline Dimensiones & Núm. de ítems & Alfa de Cronbach & Alfa de Cronbach \\
\hline Auto-aceptación & 6 & .83 & .79 \\
\hline Relaciones Positivas & 6 & .87 & .78 \\
\hline Dominio del Entorno & 6 & .71 & .63 \\
\hline Crecimiento personal & 7 & .68 & .70 \\
\hline Propósito en la vida & 6 & .83 & .80 \\
\hline Autonomía & 8 & .73 & .66 \\
\hline
\end{tabular}

Como se puede apreciar en la Tabla 1, aunque la tendencia es a la baja, la consistencia interna resultante en nuestro estudio está próxima a los valores obtenidos en el trabajo de Díez et al. (2006). Asimismo, la totalidad de las escalas, a excepción de la de Autonomía y Dominio del entorno cuya consistencia es sólo aceptable ( $\alpha=.66$ y $\alpha=.63$, respectivamente), muestran una consistencia interna moderada, con valores comprendidos entre .70 y .80. En cualquier caso, el coeficiente Alfa debe ser tomado con precaución puesto que depende de su uni-dimensionalidad (homogeneidad) (Cortina, 1993).

\subsection{Análisis estadísticos}

Primeramente, se llevó a cabo un análisis estadístico descriptivo. A continuación, se realizó un análisis inferencial para la comparación de grupos. Debido al carácter de las variables dependientes incluidas en los análisis, que en algún caso no se distribuyen de forma normal, y el tamaño reducido de los grupos, en algunos casos inferior a 30 sujetos, que denotan la falta de homocedasticidad, se han empleado pruebas estadísticas no paramétricas. Concretamente H de Kruskall-Wallis y U de Mann-Whitney. Todos estos análisis se llevaron a cabo mediante el software SPSS versión 19.0 para Macintosh.

\section{Resultados}

\subsection{Estudio Descriptivo}

En primer lugar, se representan las variables que actúan como variables independientes en el estudio. Se trató de agrupar y representar cómo se comportaron los datos recogidos de un modo ordenado atendiendo prioritariamente a las frecuencias de las variables, ya que tienen naturaleza ordinal. En aquellas tipo Likert también se incorpora la media y la desviación típica, como puede observarse en la Tabla 2. Los resultados ofrecidos desvelan información sobre una serie de cuestiones relativas al estado de salud percibido de los participantes y los hábitos saludables vinculados al ejercicio físico que afirman llevar a cabo.

En relación a la pregunta: "Recientemente, ¿diría que alguna enfermedad/es o problema/s de salud le han limitado de alguna forma sus actividades habituales?". Un total de 132 (40,2\%) participantes manifestaron que sí, mientras que 195 (59.5\%) negaron tener problemas de salud limitantes.

Por otro lado, ante la pregunta: ¿Podría indicar, si tiene dificultades para descansar lo suficiente? La mayoría, 196 participantes (59.8\%), manifestaron no tener dificultades para descansar. En el sentido opuesto, 130 participantes (39.6\%) dicen tener problemas en este sentido. 
Tabla 2. Gráficos de porcentajes
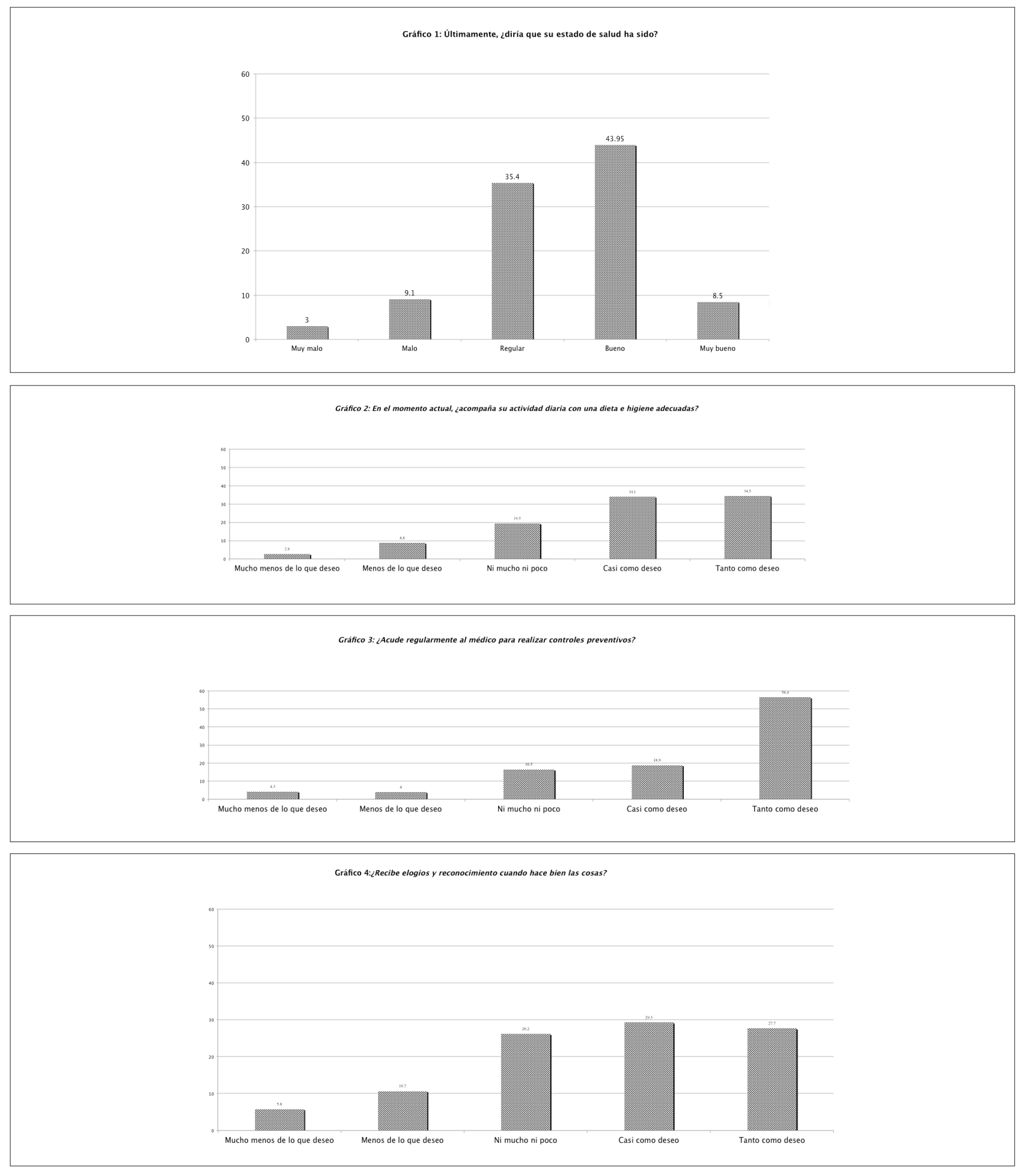

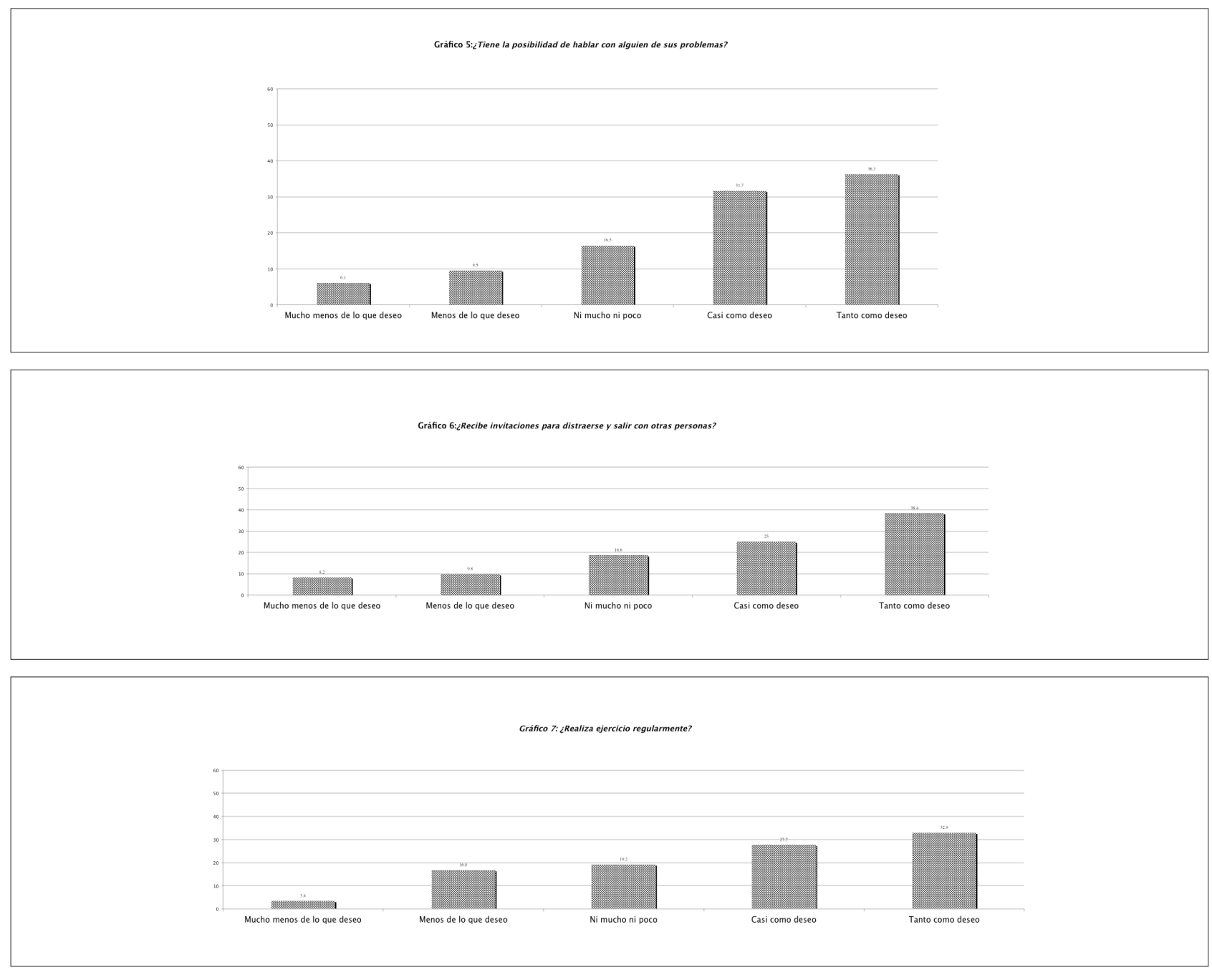

En relación a la pregunta, ¿Considera que usted cuida y vigila su salud? Una amplia mayoría afirma que sí 30 (9.1\%). Únicamente, un 8.2\% (27) de los adultos mayores del estudio manifiestan no cuidar y vigilar su estado de salud.

En cuanto a las respuestas ofrecidas a la pregunta: Durante su tiempo libre, inormalmente hace todo el ejercicio físico que desearía? Desvelan que la mayoría de los participantes (179, 54,6\%) realizan el ejercicio físico que desean. Frente a un $43 \%$ (141) que manifiestan que no.

En segundo lugar, en la Tabla 3., se muestran los estadísticos descriptivos, media y desviación típica, para cada una de las dimensiones de la Escala BP. Estas variables juegan el papel de dependientes en el estudio.

Tabla 3. Estadísticos descriptivos obtenidos en la Escala de Bienestar Psicológico de Ryff

\begin{tabular}{lccc}
\hline & N & Media & Desv. típ. \\
\hline Auto-aceptación & 328 & 4.4723 & .91328 \\
\hline Relaciones positivas & 328 & 4.2697 & .97842 \\
\hline Dominio del entorno & 328 & 4.6045 & .84295 \\
\hline Crecimiento personal & 328 & 4.2390 & .72685 \\
\hline Propósito de vida & 328 & 4.7697 & .96052 \\
\hline Autonomía & 328 & 4.2489 & .82049 \\
\hline
\end{tabular}


Tabla 4. Resultados de las pruebas estadísticas $H$ de Kruskal-Wallis y U de Mann-Whitney

Dimensiones de la escala de bienestar psicológico de RYFF Autoaceptación Relaciones Dominio Crecimiento Propósito Autonomía positivas delentorno personal de vida

\begin{tabular}{|c|c|c|c|c|c|c|c|}
\hline \multirow{3}{*}{$\begin{array}{l}\text { 1. Últimamente, } \\
\text { ¿diría que su estado } \\
\text { de salud ha sido? }\end{array}$} & Chi-cuadrado & 17.815 & 4.935 & 5.299 & 3.179 & 13.418 & 7.158 \\
\hline & gl. & 4 & 4 & 4 & 4 & 4 & 4 \\
\hline & $\rho$ & .001 & .294 & .258 & .528 & .009 & .128 \\
\hline \multirow{3}{*}{$\begin{array}{l}\text { 2. Recientemente, } \\
\text { ¿diría que alguna en- } \\
\text { fermedad/es o pro- } \\
\text { blema/s de salud le }\end{array}$} & \multicolumn{2}{|c|}{ U de Mann-Whitney 11303} & 12845 & 12749.5 & 12511.5 & 12429 & 12510 \\
\hline & Z & -1.872 & -.030 & -.144 & -.428 & -.527 & -.430 \\
\hline & $\rho$ & .061 & .976 & .886 & .669 & .598 & .667 \\
\hline
\end{tabular}

han limitado de alguna forma sus actividades habituales?

\begin{tabular}{|c|c|c|c|c|c|c|c|}
\hline \multirow{3}{*}{$\begin{array}{l}\text { 3. ¿Podría indicar, si } \\
\text { tiene dificultades para } \\
\text { descansar lo suficiente? }\end{array}$} & \multicolumn{2}{|c|}{ U de Mann-Whitney 12412,5 } & \multirow{2}{*}{$\begin{array}{l}12148 \\
-.711\end{array}$} & \multirow{2}{*}{$\begin{array}{r}12199 \\
-.651\end{array}$} & \multirow{2}{*}{$\frac{12071}{-.804}$} & \multirow{2}{*}{$\begin{array}{l}12668 \\
-.087\end{array}$} & \multirow{2}{*}{$\begin{array}{l}12715,5 \\
-.029\end{array}$} \\
\hline & Z & -.394 & & & & & \\
\hline & $\rho$ & .694 & .477 & .515 & .421 & .931 & .977 \\
\hline \multirow{3}{*}{$\begin{array}{l}\text { 4. ¿Considera que } \\
\text { usted cuida y vigila } \\
\text { su salud? }\end{array}$} & U de Mann-Whitney & 2589.5 & 3602.5 & 3344.5 & 3184 & 2561 & 3180 \\
\hline & Z & -3.128 & -.978 & -1.526 & -1.866 & -3.193 & -1.874 \\
\hline & $\rho$ & .002 & .328 & .127 & .062 & .001 & .061 \\
\hline \multirow{3}{*}{$\begin{array}{l}\text { 5. Durante su tiempo } \\
\text { libre, ¿normalmente } \\
\text { hace todo el ejercicio } \\
\text { físico que desearía? }\end{array}$} & \multicolumn{2}{|c|}{ U de Mann-Whitney10981.5 } & 12291.5 & 11524 & 12003.5 & 11065 & 12420.5 \\
\hline & Z & -1.997 & -.400 & -1.336 & -.751 & -1.898 & -.242 \\
\hline & $\rho$ & .046 & .689 & .182 & .453 & .058 & .808 \\
\hline \multirow{4}{*}{$\begin{array}{l}\text { 6. En el momento } \\
\text { actual, ¿̇acompaña su } \\
\text { actividad diaria con } \\
\text { una dieta e higiene } \\
\text { adecuadas? }\end{array}$} & Chi-cuadrado & 15.908 & 2.443 & 7.824 & 2.738 & 6.169 & 13.746 \\
\hline & gl. & 4 & 4 & 4 & 4 & 4 & 4 \\
\hline & $\rho$ & .003 & .655 & .098 & .603 & .187 & .008 \\
\hline & & & & & & & \\
\hline \multirow{3}{*}{$\begin{array}{l}\text { 7. ¿Acude regularmente } \\
\text { al médico para realizar } \\
\text { controles preventivos? }\end{array}$} & Chi-cuadrado & 19.817 & 10.704 & 20.936 & 10.175 & 19.335 & 24.910 \\
\hline & gl. & 4 & 4 & 4 & 4 & 4 & 4 \\
\hline & $\rho$ & .001 & .030 & .000 & .038 & .001 & .000 \\
\hline \multirow{3}{*}{$\begin{array}{l}\text { 8. ¿Recibe elogios y re- } \\
\text { conocimiento cuando } \\
\text { hace bien las cosas? }\end{array}$} & Chi-cuadrado & 20.776 & 16.195 & 29.500 & 13.970 & 19.905 & 12.683 \\
\hline & gl. & 4 & 4 & 4 & 4 & 4 & 4 \\
\hline & $\rho$ & .000 & .003 & .000 & .007 & .001 & .013 \\
\hline \multirow{3}{*}{$\begin{array}{l}\text { 9. ¿Tiene la posibilidad } \\
\text { de hablar con alguien } \\
\text { de sus problemas? }\end{array}$} & Chi-cuadrado & 12.754 & 25.521 & 20.560 & 7.990 & 16.800 & 5.723 \\
\hline & gl. & 4 & 4 & 4 & 4 & 4 & 4 \\
\hline & $\rho$ & .013 & .000 & .000 & .092 & .002 & .221 \\
\hline \multirow{3}{*}{$\begin{array}{l}\text { 10. ¿'Recibe invitaciones } \\
\text { para distraerse y salir } \\
\text { con otras personas? }\end{array}$} & Chi-cuadrado & 21.589 & 26.223 & 32.020 & 12.885 & 26.153 & 10.552 \\
\hline & gl. & 4 & 4 & 4 & 4 & 4 & 4 \\
\hline & $\rho$ & .000 & .000 & .000 & .012 & .000 & .032 \\
\hline \multirow{3}{*}{$\begin{array}{l}\text { 11. ¿'Realiza ejercicio re- } \\
\text { gularmente? }\end{array}$} & Chi-cuadrado & 12.667 & 1.036 & 13.750 & 2.968 & 8.060 & 7.003 \\
\hline & gl. & 4 & 4 & 4 & 4 & 4 & 4 \\
\hline & $\rho$ & .013 & .904 & .008 & .563 & .089 & .136 \\
\hline
\end{tabular}




\subsection{Análisis inferencial}

Este estudio tuvo por objetivo comprobar si el estado de salud percibido y los hábitos saludables producen diferencias en las dimensiones de bienestar psicológico. Es decir, si ese estado de salud está relacionado con el bienestar psicológico. Para ello, se partió del planteamiento de varias hipótesis para cada una de las pruebas realizadas.

Los resultados de las pruebas estadísticas realizadas se resumen en la siguiente tabla. Se han marcado en negrita los resultados significativos, es decir, aquellos cuya probabilidad asociada es inferior a .05.

Hipótesis 1. Existen diferencias en los resultados de las dimensiones de la escala BP en función de la percepción del estado de salud.

En primer lugar, para determinar si existían diferencias en los resultados obtenidos en las dimensiones de la escala BP, en función de la percepción del estado de salud que cuenta con cinco grupos (muy malo, malo, regular, bueno y muy bueno), se utilizó la prueba no paramétrica $\mathrm{H}$ de Kruskal-Wallis. Esta herramienta de contraste es adecuada para la comparación de más de dos grupos no homogéneos, o cuando las variables dependientes no tienen distribución normal. Los resultados muestran que sí existen diferencias estadísticamente significativas en las puntuaciones de Autoaceptación ( $p=.001$ ) y Propósito de vida ( $p=.009)$. La tendencia encontrada es que las personas que afirman que su estado de salud ha sido muy malo tienden a presentar resultados más elevados en Auto-aceptación y Propósito en la vida que aquellos que lo consideran bueno o muy bueno. Únicamente es posible afirmar tendencia en los resultados porque la prueba de Kruskal-Wallis no permiten realizar contrastes posteriores.

Hipótesis 2. Existen diferencias en los resultados de las dimensiones de la escala BP en función de la apreciación de tener enfermedad/es o problema/s de salud que les limitan de alguna forma sus actividades habituales.

Se analizó la influencia sobre las dimensiones del bienestar psicológico subjetivo de la afirmación o negación de tener de alguna enfermedad/es o problema/s de salud que limita de alguna forma sus actividades habituales. Se aplicó la prueba U de Mann-Whitney, adecuada para comparar los resultados de dos grupos. No se encontró ningún efecto significativo en las dimensiones del BP.

Hipótesis 3. Existen diferencias en los resultados de las dimensiones de la escala BP en función de la percepción de tener o no dificultades para descansar lo suficiente.

La prueba U de Mann-Whitney calculada muestra que no existen diferencias estadísticamente significativas en las dimensiones del BP en función de esta variable.

Hipótesis 4. Existen diferencias en los resultados de las dimensiones de la escala BP en función de la consideración de cuidar o no su estado de salud.

Tras la realización de la prueba de contraste U de Mann-Whitney, se constata que aquellos que consideran que cuidan y vigilan su salud obtienen puntuaciones más altas en las dimensiones de Autoaceptación ( $(\mathrm{p}=.002$ ) y Propósito en la vida $(\mathrm{p}=.001)$, que aquellos que consideran que no la cuidad y vigilan. En este caso, al contar con dos grupos únicos, si pueden afirmarse diferencias estadísticamente significativas entre ellos. 
Hipótesis 5. Existen diferencias en los resultados de las dimensiones de la escala BP en función de la realización de todo el ejercicio físico que desean durante su tiempo libre.

Se aplicó la prueba $\mathrm{H}$ de Kruskal-Wallis y se encontraron diferencias significativas en las puntuaciones de la dimensión de Autoaceptación ( $p=.046$ ). De tal modo, aquellos que afirman que durante su tiempo libre realizan el ejercicio físico que desean obtienen puntuaciones altas en la dimensión de Autoaceptación frente a los que los que no lo realizan.

Hipótesis 6. Existen diferencias en los resultados de las dimensiones de la escala BP en función de si acompañan su actividad diaria con una dieta e higiene adecuadas.

Se utilizó la prueba $\mathrm{H}$ de Kruskal-Wallis y se han encontrado diferencias en las dimensiones de Autoaceptación ( $p=.003$ ) y Autonomía ( $p=.008$ ). En ambas dimensiones, aquellos que afirman que acompañan la actividad diaria con una dieta e higiene adecuadas tanto como desean obtienen las puntuaciones más altas. La tendencia es creciente, en ambas dimensiones las puntuaciones aumentan a medida que se afirma hacer tanto como se desea.

Hipótesis 7. Existen diferencias en los resultados de las dimensiones de la escala BP en función de si afirman acudir regularmente al médico para realizar controles preventivos.

Se aplicó la prueba $\mathrm{H}$ de Kruskal-Wallis y revela que la valoración de la variable acudir regularmente al médico para realizar controles preventivos produce diferencias significativas en todas las dimensiones de bienestar psicológico. Las puntuaciones más altas son las logradas por aquellos que afirman realizar tantos controles como desean.

Hipótesis 8. Existen diferencias en los resultados de las dimensiones de la escala BP en función de si afirman recibir elogios y reconocimientos cuando hacen bien las cosas.

Se ha empleado la prueba $\mathrm{H}$ de Kruskal-Wallis y los resultados muestran que existen diferencias significativas en las puntuaciones de todas las dimensiones de bienestar psicológico provocadas por la variable recibir elogios y reconocimiento. De tal modo, aquellos adultos mayores que afirman que no reciben tantos elogios como desean tienden a tener valores inferiores de las distintas variables de bienestar psicológico. De forma opuesta, los que contestaron que recibían tantos elogios como deseaban obtuvieron las puntuaciones más altas.

Hipótesis 9. Existen diferencias en los resultados de las dimensiones de la escala BP en función de si afirman tener la posibilidad de hablar con alguien de sus problemas.

Se aplicó la prueba $\mathrm{H}$ de Kruskal-Wallis y se han hallado diferencias significativas en las dimensiones de Autoaceptación ( $p=.013$ ), Relaciones positivas ( $p=.000)$, Dominio del entorno ( $p=.000)$ y Propósito en la vida ( $p=.002)$. La tendencia es similar en todas las variables, se obtienen puntuaciones más altas a medida que se habla de los problemas con alguien tanto como se desea.

Hipótesis 10. Existen diferencias en los resultados de las dimensiones de la escala BP en función de si reciben invitaciones para salir y distraerse con otras personas.

La prueba $\mathrm{H}$ de Kruskal-Wallis revela diferencias estadísticamente significativas en todas las dimensiones de bienestar psicológico en función la recepción de invitaciones para salir y distraerse. La ten-

[ 164 ] ÁNGEL DE-JUANAS OLIVA, MARÍA ROSARIO LIMÓN MENDIZÁBAL, ENRIQUE NAVARRO ASENSIO SIPS - PEDAGOGIA SOCIAL. REVISTA INTERUNIVERSITARIA [1139-1723 (2013) 22, 153-168] TERCERA ÉPOCA 
dencia encontrada es que a medida que se reciben tantas invitaciones como se desean para salir y divertirse obtienen un mayor bienestar psicológico. Por tanto, aquellos que afirman que se distraen menos de lo que desean obtienen puntuaciones más bajas en la totalidad de las dimensiones de bienestar psicológico.

Hipótesis 11. Existen diferencias en los resultados de las dimensiones de la escala BP en función de si consideran que realizan ejercicio regularmente.

Se aplicó la prueba $\mathrm{H}$ de Kruskal-Wallis y se han encontrado diferencias significativas en Autoaceptación $(p=.013)$ y Dominio del entorno $(p=.008)$ producidos por la realización de ejercicio físico de forma regular. La tendencia muestra que las puntuaciones en ambas dimensiones son mayores a medida que se realiza todo el ejercicio que se desea.

\section{Discusión}

A la vista de los resultados de otros estudios, los adultos mayores suelen reportar mayores niveles de bienestar subjetivo que las personas más jóvenes (Plagnol y Easterlin, 2008). Partiendo de esta evidencia, en el presente trabajo, se ha puesto de relieve que el estado de salud percibido y los hábitos saludables como una adecuada dieta e higiene o la actividad física influyen en cómo se sienten los adultos mayores españoles consigo mismos. De tal manera, diversos trabajos han hallado que la salud percibida es un importante predictor del bienestar psicológico en edades avanzadas (Kirby, Coleman y Daley, 2004, Stone et al., 2010, Yang, 2008,).

Asimismo, se constata que aquellos que afirman realizar ejercicio físico tienden a mostrar una mayor habilidad personal para elegir o crear entornos favorables y satisfacer los deseos y necesidades propias. Siendo esta una característica de funcionamiento positivo. Al respecto, numerosas investigaciones han demostrado la influencia de la práctica regular de actividad física en la mejora del bienestar de las personas mayores. Estos trabajos han encontrado que las personas que realizan actividad física independientemente del tipo de ejercicio practicado obtienen mejoras en su estado de ánimo (Jiménez et al., 2006). Esto concuerda con otros estudios en los que incluso se asevera que a mayor bienestar psicológico más disfrute por la actividad física realizada (e.g., Gracia y Marco, 2000).

Por otro lado, se ha observado que la aparición de enfermedades que inciden en las actividades habituales no influye negativamente en los niveles de bienestar subjetivo, estos resultados coinciden con los obtenidos en otras investigaciones (Baltes y Carstensen, 2003).

En otro sentido, en los ítems relacionados con las relaciones sociales y el apoyo social percibido, los resultados evidencian que los mayores que no salen con otras personas tienen menos posibilidades de hablar con otros sobre sus problemas. Se distraen menos de lo que desean y reciben menos elogios y obtienen puntuaciones más bajas en la totalidad de las dimensiones de bienestar psicológico. Como era previsible, las relaciones interpersonales actúan sobre el bienestar psicológico de un modo positivo, así lo reflejan los estudios de Ferguson y Goodwin (2010) y Friedman (2012).

Aunque la base del presente estudio es pequeña y los hallazgos deben tomarse con precaución, convendría tener en cuenta los resultados para avanzar hacia una concepción integral de la persona mayor que deberá concretarse en una mayor coordinación institucional e intersectorial, o lo que es lo mismo, que el envejecimiento sea tema de interés para todas las áreas de las políticas públicas. Para lo que se requiere, a su vez, modificar progresivamente los estereotipos negativos que en nuestro contexto cultural actual pesan sobre la vejez y las personas mayores. Sin una actitud positiva por parte de los responsables políticos, de las propias personas mayores y de la sociedad en su conjunto será muy difícil lograr cambios (Pérez de Guzmán, 2005). Del mismo modo, se debe intervenir sobre el actual modelo de trabajo de las instituciones más centradas en "hacer para" que en "hacer con", porque ello 
facilitará una mayor implicación (frente a la mera recepción pasiva) de las personas mayores en la construcción de su realidad.

Finalmente, se ha de contribuir al desarrollo personal de los adultos mayores facilitando una mayor implicación y participación de los mismos en los procesos comunitarios (Lirio, Alonso y Herranz, 2009). Asimismo, ayudar a las personas de edad avanzada a consolidar hábitos de ocio activos y saludables que sean una fuente de estimulación física, cognitiva y social que, además, proporcione satisfacción y objetivos vitales (Pérez Serrano, 2005).

\section{Referencias bibliográficas}

Gracia, M. y Marco, M. (2000).Efectos psicológicos de la actividad física en personas mayores.Psicothema, 12(2), $285-292$.

Cortina, J. M. (1993). What is coefficient alpha? An examination of theory and aplications. Journal of Educational Psychology, 78(1), 98-104.

Kelloway, E. K., Catano, U. M. y Soutwell, R. R. (1992). The construct validity of union commitment: Development and dimensionality of a shorter scale. Journal of Occupational of Organizational Psychology, 65, 197-211.

Díaz, D., Rodríguez-Carvajal, R., Blanco, A., Moreno-Jiménez, B., Gallardo, I., Valle,C. y Van Dierendonck, D. (2006). Adaptación Española de las escalas de Bienestar Psicológico. Psicothema,18 (3), 572-577.

Ferguson, S. y Googwin, A. (2010). Optimism and Well-Being in Older Adults: The Mediating Role of Social Support and Perceived Control. The International Journal of Aging and Human Development, 71, 43-68.

Fernández Ballesteros, R. (1992). Dimensiones de la evolución de la calidad de vida. Madrid: Universidad Autónoma.

Fernández, J. D. y Limón, Ma. R. (2012). El arte de envejecer con humor. Málaga: Aljibe.

George, L. K. (2006). Perceived quality of life. In R.H. Binstock, L. K. George (editors), Handbook of aging and the social sciences, sixth edition, (pp. 320-336) San Diego, CA: Academic Press.

George, L. K. (2010). Still Happy After All These Years: Research Frontiers on Subjective Well-being in Later Life. The Journals of Gerontology: Series B, 65B(3), 331-339.

Gonçalves Marques, E. M. (2012). Envelhecimento no Concelho da Guarda. Un estudo sobre qualidade de vida. Tesis Doctoral. Universidad Pontificia de Salamanca.

IMSERSO (2010). Informe Anual 2010. Madrid: IMSERSO.

Instituto Nacional de Estadística. Encuesta Nacional de Salud, Cuestionario de adultos, 2006. Madrid: Ministerio de Sanidad, Servicios Sociales e Igualdad. Recuperado de http://www.msps.es/estadEstudios/estadisticas/encuestaNacional/ encuestaNac2006/ENS_06_Adultos_definitivo.pdf

Instituto Nacional de Estadística (2009). Encuesta Europea de Salud, 2009. Madrid: Ministerio de Sanidad, Servicios Sociales e Igualdad. Recuperado de http://www.ine.es/metodologia/t15/t153042009cues.pdf

Jiménez, M., Martínez, P., Miró E. y Sánchez, A. (2006). Bienestar psicológico y hábitos saludables: ¿están asociados a la práctica de ejercicio físico? International Journal of Clinical and Health Psycholgy, 8(1), 185-2O2.

Keyes, C., Ryff, C. y Shmotkin, D. (2002). Optimizing well-being: theempirical encounter of two traditions. Journal of Personality and Social Psychology, 82, 1007-1022.

Kirby, S.E., Coleman, P. G. y Daley, D. (2004). Spirituality and well-being in frail and non-frail older adults. Journal of Gerontology: Psychological Sciences, 59(3), 123-129.

Lehr, Ú. (2008). La longevidad, un reto para el individuo y la sociedad. En Foro de la Sociedad Civil sobre Envejecimiento, celebrado en León, noviembre 2007. Madrid: IMSERSO.

Lirio, J., Alonso, D. y Herránz, I. (2009). Envejecer participando. El proyecto "Entre mayors". Una experiencia de investigación acción. Castilla-La Mancha: Miño y Dávila.

Limón Mendizábal, Ma. R. (2011). Nuevos retos de la educación para la salud en las personas mayores. En R. Perea Quesada, Educación para la salud y calidad de vida, pp. 149-177. Madrid: Díaz de Santos.

Montiel, P. y Merino, A. (2011). Beneficios psico-sociales de la actividad física. Jornada celebrada en el IMSERSO el día24 de febrero. Madrid: Ministerio de Sanidad y Política Social.

[ 166 ] ÁNGEL DE-JUANAS OLIVA, MARÍA ROSARIO LIMÓN MENDIZÁBAL, ENRIQUE NAVARRO ASENSIO SIPS - PEDAGOGIA SOCIAL. REVISTA INTERUNIVERSITARIA [1139-1723 (2013) 22, 153-168] TERCERA ÉPOCA 
Mora, F. (2009). Claves para envejecer con éxito. Revista 60 y Más, 285, 14-17. Madrid: IMSERSO.

Naciones Unidas (2002). Informe de la Segunda Asamblea Mundial sobre el Envejecimiento. Nueva York: Naciones Unidas.

Organización Mundial de la Salud (2001). Ageing and Health. Acheiving health across the spam. Geneve: OMS.

Pérez de Guzmán Puya, M. V. (Coord.) (2005). Organización y gestión. Agentes al servicio de los mayores. Madrid: Universitas.

Pérez Serrano, G. (Coord.) (2005). Intervención y desarrollo integral en personas mayores. Madrid: Universitas.

Plagnol, A. C. y Easterlin, R. A. (2008). Aspirations, attainments, and satisfaction: Life cycle differences between American women and men. Journal of Happiness Studies, 9, 601-619.

Rodríguez-Artalejo, F. (2011). Envejecer satisfactoriamente. Revista Española de Geriatría y Gerontología, 46(1), 1-5.

Rubio Florido, I. (2012). El tiempo de ocio de los cuidadores familiares: Su relación con la salud y la percepción de carga. Tesis doctoral. Bilbao: Universidad de Deusto.

Ryan, R. M. y Deci, E. L. (2001). To be happy or to be self-fulfilled: A review of Research on hedonic and eudaemonic wellbeing. In S. Fiske (Ed.), Annual Review of Psychology (pp. 141-166). Palo Alto, CA: Annual Reviews, Inc.

Ryff, C. D. (1989a). In the eye of the beholder: views of psychological well-being among middle-aged and older adults. Psychol Aging, 4, 195-210.

Ryff, C. D. (1989b). Happiness is Everything, or Is It? Explorations on the Meaning of Psychological Well-Being.Journal of Personality and Social Psychology, 57(6), 1069-1081.

Ryff, C.D. y Keyes, C. L. (1995). The structure of Psychological Well-Being Revisited.Journal of Personality and Social Psychology, 69 (4), 719-727.

Schalock, R. L. y Verdugo, M. A. (2003).Calidad de vida - Manual para profesionales de la educación, salud y servicios sociales. Madrid: Alianza Editorial.

Stone, A., Schwartz, J. E., Broderick, J. E. y Deaton, A. (2010).A snapshot of the age distribution of psychological well-being in the United States. PNAS, 107(22), 1-6.

UNFPA (2011). Estado de la población mundial 2011. Nueva York: UNFPA.

Yang, Y. (2008). Social inequalities in happiness in the United States, 1972 to 2004: An age-period-cohort analysis. American Sociological Review, 73, 204-236.

\section{Notas}

${ }^{1}$ Esta investigación ha sido financiada por la Agencia Española de Cooperación al Desarrollo AECID. Título del proyecto: Intervención educativa en contextos sociales. Referencia AP/036665/11

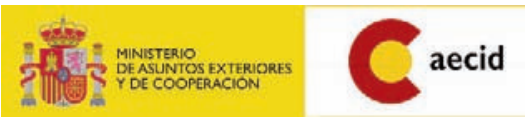

2 World Health Organization Quality of Life.

3 Véase el cuestionario de adultos que se puede encontrar en: http://www.msps.es/estadEstudios/estadisticas/encuestaNacional/encuesta2006.htm

4 Puede encontrarse más información en:

http://www.ine.es/jaxi/menu.do?type=pcaxis\&path=\%2Ft15/p420\&file=inebase

\section{Dirección de los autores}

Ángel De-Juanas Oliva. Universidad Nacional de Educación a Distancia. Departamento de Teoría de la Educación y Pedagogía Social. Facultad de Educación. C/ Juan del Rosal, 14. 28030 - Madrid.

María Rosario Limón Mendizábal. Universidad Complutense de Madrid. Departamento de Teoría e Historia de la Educa- 
ción. Avda. de Séneca, 2. Ciudad Universitaria. 28040 Madrid

Enrique Navarro Asencio. Universidad Internacional de la Rioja. Departamento de Educación. C/ Albacete, 5,1 planta,Ala Este, 28027, Madrid

Correo electrónico: adejuanas@edu.uned.es, mrlimonm@edu.ucm.es, enrique.navarro@unir.net

Fecha de recepción del artículo: 29.1.2013

Fecha de revisión del artículo: 4.3.2013

Fecha de aceptación final: 15.4.2013

\section{Cómo citar este artículo}

De-Juanas Oliva, A., Limón Mendizábal, M. R. y Navarro Asencio, E. (2013). Análisis del bienestar psicológico, estado de salud percibido y calidad de vida en personas adultas mayores. Pedagogía Social. Revista Interuniversitaria, 22, pp. 153-168.

[ 168 ] ÁNGEL DE-JUANAS OLIVA, MARÍA ROSARIO LIMÓN MENDIZÁBAL, ENRIQUE NAVARRO ASENSIO SIPS - PEDAGOGIA SOCIAL. REVISTA INTERUNIVERSITARIA [1139-1723 (2013) 22, 153-168] TERCERA ÉPOCA 